Meta

Journal des tradlucteurs

Translators' Journal

\title{
Du walkie-talkie et de la difficulté de déraciner les emprunts
}

\section{Robert Dubuc}

Volume 22, numéro 4, décembre 1977

URI : https://id.erudit.org/iderudit/002637ar

DOI : https://doi.org/10.7202/002637ar

Aller au sommaire du numéro

Éditeur(s)

Les Presses de l'Université de Montréal

ISSN

0026-0452 (imprimé)

1492-1421 (numérique)

Découvrir la revue

Citer cet article

Dubuc, R. (1977). Du walkie-talkie et de la difficulté de déraciner les emprunts. Meta, 22(4), 270-272. https://doi.org/10.7202/002637ar d'utilisation que vous pouvez consulter en ligne.

https://apropos.erudit.org/fr/usagers/politique-dutilisation/ 


\section{DROBLIEMES ET SOLUTIONS}

\section{DU WALKIE-TALKIE ET DE LA DIFFICULTÉ DE DÉRACINER LES EMPRUNTS}

En électroacoustique, le mot walkie-talkie désigne un appareil émetteur-récepteur portatif servant à des communications sur de faibles distances. En pratique, aucun équivalent ne s'est vraiment imposé jusqu'ici. MOBILOPHONE qui, à un moment donné, a semblé pouvoir s'imposer dans ce sens, a connu une spécialisation qui l'écarte désormais de la liste des équivalents possibles. Selon le Dictionnaire de l'électronique de Larousse ${ }^{1}$, le mot ne s'applique qu'aux émetteurs-récepteurs installés dans les véhicules: des espèces de $\mathrm{CB}^{2}$ avant la lettre.

Parmi les autres équivalents recueillis, il convient de noter ÉMETTEURRÉCEPTEUR PORTATIF ${ }^{3}$, RADIOTÉLÉPHONE PORTATIF ${ }^{\star}$, INTERPHONE PORTATIF ${ }^{5}$, TOP-TOC ${ }^{6}$, COMBINÉ PORTATIF ${ }^{7}$. On peut se poser la question: y a-t-il lieu de remplacer l'emprunt et, le cas échéant, par quel équivalent?

Pour répondre à cette question, nous allons étudier chaque expression en fonction de quatre critères qui semblent favoriser l'accréditation d'un terme dans l'usage : fréquence, maniabilité, adéquation, motivation.

1. J.-F. Arnaud, Dictionnaire de l'électronique, Paris, Larousse, coll. ¿ Dictionnaire de l'homme du XXe siècle », au mot mobilophone.

2. $\mathrm{CB}$, abréviation de Citizen's Band, désigne des appareils émetteurs-récepteurs fonctionnant sur des fréquences réservées aux communications privées. Ces appareils connaissent présentement une très grande vogue en Amérique du Nord. On les désigne parfois par l'acronyme FRECOP (fréquences réservées aux communications privées).

3. H. Piraux, Dictionnaire anglais-français des termes relatifs à l'électronique et à l'électrotechnique, Paris, Eyrolles, 11e éd., 1975, au mot walkie-talkie.

4. G. Belle-İsle, Dictionnaire technique général, anglais-français, Québec, Belisle édit., 1965, au mot walkie-talkie.

5. Comité de linguistique, Fiches, Montréal, Société Radio-Canada, 1965, rubrique walkietalkie.

6. Comité d'étude des termes techniques français, $118^{\mathrm{e}}$ réunion, 1970.

7. Gérard Proulx, Dictionnaire anglais-français, électronique et télécommunications, Québec, Belisle édit., 1959; au mot walkie-talkie. 
La fréquence est fonction de l'utilisation du terme par l'ensemble des sujets parlant soit la langue générale, soit une langue de spécialité. La maniabilité tient à la brièveté du terme, à sa possibilité d'intégration dans le système morphologique du français. L'adéquation souligne la convenance entre le signifiant et le signifié qui se manifeste surtout par l'absence d'ambiguitté. Enfin, la motivation est cette propriété que peut avoir un terme de laisser transparaître sa signification. Elle peut être d'ordre morphologique : étymologie, ou sémantique : analogie de forme ou de fonction.

Prenons maintenant chacun de ces termes isolément :

WALKIE-TALKIE. Ce terme est un emprunt intégral à l'anglais. Il figure au Petit Larousse (1977), au Dictionnaire encyclopédique Quillet (1968) è au Grand Larousse encyclopédique $(1960+)$. On ne le trouve cependant ni aux Robert (grand et petit), ni dans le Bordas ${ }^{8}$, ni dans le Lexis $^{9}$. Les dictionnaires techniques bilingues (Piraux, Malgorn, Belle-Isle) n'accréditent pas cet emprunt, sauf Proulx ${ }^{10}$ qui le donne comme synonyme de combiné portatif. Pour qui ignore l'anglais, ce mot n'est nullement motivé. Son intégration phonétique et graphique est difficile, pourtant ces défauts ne l'ont pas empêché de se répandre suffisamment pour figurer dans certains dictionnaires usuels.

ÉMETTEUR-RÉCEPTEUR PORTATIF. Cet équivalent, donné par Piraux, est plutôt une description qu'une étiquette. L'expression n'est pas vraiment lexicalisée et ne figure comme telle dans aucun des dictionnaires unilingues consultés. L'expression est très motivée, mais lourde à manier. Aussi semble-t-elle d'un emploi rare.

RADIOTÉLÉPHONE PORTATIF. Le radiotéléphone établit une liaison téléphonique duplex le plus souvent à grande distance au moyen des ondes radioélectriques ${ }^{11}$. Le système simple émetteur-récepteur du walkie-talkie ne saurait, sans risque de confusion, être assimilé au radiotéléphone. En ce sens, l'expression radiotéléphone portatif ne semble pas adéquate.

INTERPHONE PORTATIF. L'interphone est un système électronique de communications intérieures à plusieurs directions, utilisant des transducteurs réversibles qui servent à la fois comme microphone et haut-parleur ${ }^{12}$. C'est par analogie de fonction qu'on pourrait étendre le sens du mot interphone au walkie-talkie. L'expression est maniable, facile à comprendre et facilement lexicable.

TOP-TOC. Création plaisante du Comité d'étude des termes techniques français, cette expression n'a jamais pris le chemin de l'usage malgré sa brièveté, son caractère sonore d'onomatopée. Il semble que sa motivation soit trop arbitraire. Elle risque d'être une création mort-née.

\footnotetext{
8. M. Davau, M. Cohen et M. Lallemand, Dictionnaire du français vivant, Paris, Bordas, $1972,1277 \mathrm{p}$

9. Voir Grand Larousse encyclopédique, sub verbis.

10. Gérard Proulx, Dictionnaire anglais-français, électronique et télécommunications.

11. Voir Grand Larousse encyclopédique, sub verbis.

12. Voir H. Piraux, Dictionnaire général d'acoustique et d'électroacoustique, Paris, Eyrolles, 1964 , sub verbis.
} 
COMBINÉ PORTATIF. Ici encore nous sommes en présence d'une extension sémantique par analogie. Le combiné est cette partie de l'appareil téléphonique qui regroupe l'émetteur et le récepteur. L'adjectif portatif implique la liaison radioélectrique. L'expression pourrait être viable : elle est maniable, motivée, mais elle reste peu usitée.

TABLEAU DE PONDÉRATION DES EXPRESSIONS CONCURRENTES D'APRES LES CRITĖRES DE SÉLECTION DÉJẢ PRÉCITÉS

\begin{tabular}{lccccc}
\hline Termes & Fréquence & Maniabilité & Adéquation & Motivation & Total \\
\hline $\begin{array}{l}\text { Walkie-talkie } \\
\begin{array}{l}\text { Emetteur-récepteur } \\
\text { portatif }\end{array}\end{array} \quad 9$ & 5 & 10 & 0 & 24 \\
$\begin{array}{l}\text { Radiotéléphone } \\
\text { portatif }\end{array}$ & 5 & 2 & 10 & 10 & 27 \\
$\begin{array}{l}\text { Interphone } \\
\text { portatif }\end{array}$ & 1 & 4 & 3 & 6 & 14 \\
$\begin{array}{l}\text { Top-toc } \\
\text { Combiné portatif }\end{array}$ & 1 & 5 & 6 & 8 & 20 \\
\hline
\end{tabular}

\section{Conclusion}

Cette quantification numérique des divers critères de sélection ne peut être interprétée de façon absolue. Mais elle fournit des indications moins subjectives sur l'appréciation des termes en présence. Par exemple, si l'on a tendance à ne considérer que la maniabilité, un terme comme top-toc serait d'emblée retenu. En faisant intervenir les autres critères, le mot prend une place beaucoup plus relative.

Il ne faudrait pas conclure que l'expression qui obtient l'indice numérique le plus élevé est nécessairement celle qui s'imposera dans l'usage. C'est au mieux une évaluation de probabilités. L'usage est trop capricieux pour se laisser conditionner par des critères de sélection.

ROBERT DUBUC 